\title{
Genome subtraction for novel target definition in Salmonella typhi
}

\author{
Bhawna Rathi*, Aditya N. Sarangi, Nidhi Trivedi
}

Biomedical Informatics Centre, Sanjay Gandhi Postgraduate Institute of Medical Sciences, Lucknow 226014, India; Bhawna Rathi - Email: bhawna_rathi3@rediffmail.com; *Corresponding author

\begin{abstract}
:
Large genomic sequencing projects of pathogens as well as human genome leads to immense genomic and proteomic data which would be very beneficial for the novel target identification in pathogens. Subtractive genomic approach is one of the most useful strategies helpful in identification of potential targets. The approach works by subtracting the genes or proteins homologous to both host and the pathogen and identify those set of gene or proteins which are essential for the pathogen and are exclusively present in the pathogen. Subtractive genomic approach is employed to identify novel target in salmonella typhi. The pathogen has 4718 proteins out of which 300 are found to be essential ("indispensable to support cellular life") in the pathogen with no human homolog. Metabolic pathway analyses of these 300 essential proteins revealed that 149 proteins are exclusively involved in several metabolic pathway of S. typhi. 8 metabolic pathways are found to be present exclusively in the pathogen comprising of 27 enzymes unique to the pathogen. Thus, these 27 proteins may serve as prospective drug targets. Sub-cellular localization prediction of the 300 essential proteins was done which reveals that 11 proteins lie on the outer membrane of the pathogen which could be probable vaccine candidates.
\end{abstract}

Received May 07, 2009; Revised August 24, 2009; Accepted September 11, 2009; Published October 11, 2009

Keywords: Subtractive genomics approach, proteome, drug targets.

Background:

The availability of large amount of genomic data generated by the microbial genomes and the human genome project has revolutionized the field of drug-discovery against threatening human pathogens [1]. These large sets of genomic data are useful in identification and characterization of the novel therapeutic targets and virulent factors prevalent in the pathogens. Subtractive genomic strategy is developed by assuming that the novel targets identified in the pathogen should be essential for the pathogen that is it should be involved in the replication, survival and a important component of various metabolic pathways and mechanisms occurring in the pathogen while at the same time should be absent on the host that is human and should have no homolog in human, so that when a drug or a lead compound is designed considering the potential target it should only be against the mechanism and functionality of the pathogen not the host. Subtractive genomics has been successfully used by authors to locate novel drug targets in Pseudomonas aeruginosa [2]. The work has been effectively complemented with the compilation of the Database of Essential Genes (DEG) for a number of pathogenic microorganisms [3]. The current studies make use of the subtractive genomics approach and DEG to analyze the complete genome of Salmonella typhi to search for potential vaccine candidates which would possibly lie on the surface membrane of the pathogen and drug targets.

Salmonella enterica serovar typhi is a human-specific gram-negative pathogen causing enteric typhoid fever, a severe infection of the reticuloendothelial system [4], [5], [6]. It has two strains CT18 (multiple drug resistant) [7] and Ty with a complete proteome of 4718 proteins. Worldwide, typhoid fever affects roughly millions of people annually, causing deaths. Infection of $S$. typhi leads to the development of typhoid, or enteric fever. This disease is characterized by the sudden onset of a sustained and systemic fever, severe headache, nausea, and loss of appetite. Other symptoms include constipation or diarrhea, enlargement of the spleen, possible development of meningitis, and/or general depression. Untreated typhoid fever cases result in mortality rates ranging from $12-30 \%$ while treated cases allow for $99 \%$ survival. The early administration of antibiotic treatment has proven to be highly effective in eliminating infections, but indiscriminate use of antibiotics has led to the emergence of multidrug-resistant strains of $S$. enterica serovar Typhi [8]. Chloramphenicol was the drug for the treatment of this infection till plasmid mediated chloramphenicol resistance was encountered [9]. Following this ciprofloxacin became the mainstay of treatment being a safer and more effective drug than Chloramphenicol but after clinical resistance to treatment with ciprofloxacin in the patients suffering from enteric fever, the choice left now is an expensive drug like ceftriaxone or cefexime.[10]. Resistance against ceftriaxone have been reported to CDC (Centre for Drug Control) [11] mild to moderate side effects have been shown for ceftriaxone. The novel targets identified by us using subtractive genomics will help enable understanding the biology of the pathogen to provide a more cost effective medication.

\section{Methodology:}

The systematic identification and characterization of potential targets in salmonella typhi is illustrated in Figure 1.

\section{Retrieval of proteomes of host and pathogen:}

The complete proteome of Salmonella typhi were retrieved from SwissProt [12] and protein sequences of Homo sapiens were downloaded from NCBI [13]. The Database of Essential genes was accessed from its location http://tubic.tju.edu.cn/deg/.

Identification of essential proteins in S. typhi:

The $S$. typhi proteins were purged at $60 \%$ using CD-HIT [14] to identify the paralogs or duplicates proteins within the proteome of S.typhi. The paralogs are excluded and the remaining sets of protein were subjected to BlastP against Homo sapiens protein sequences with the expectation value (E-value) cutoff of $10^{-4}$. The resultant dataset obtained were with no homologs in Homo sapiens. BLASTP analysis was performed for the non homologous protein sequences of $S$. typhi against DEG with E-value cutoff score of $10^{-100}$. A minimum bitscore cut-off of 100 was used to screen out genes that appeared to represent essential genes. The protein sequences obtained are non homologous essential proteins of S.typhi.

\section{Metabolic pathway analysis:}

Metabolic pathway analysis of the essential proteins of S. typhi was done by KAAS server at KEGG for the identification of potential targets. KAAS (KEGG Automatic Annotation Server) provides functional annotation of genes by BLAST comparisons against the manually curated KEGG GENES database. The result contains KO (KEGG Orthology) assignments and automatically generated KEGG pathways. [15] 


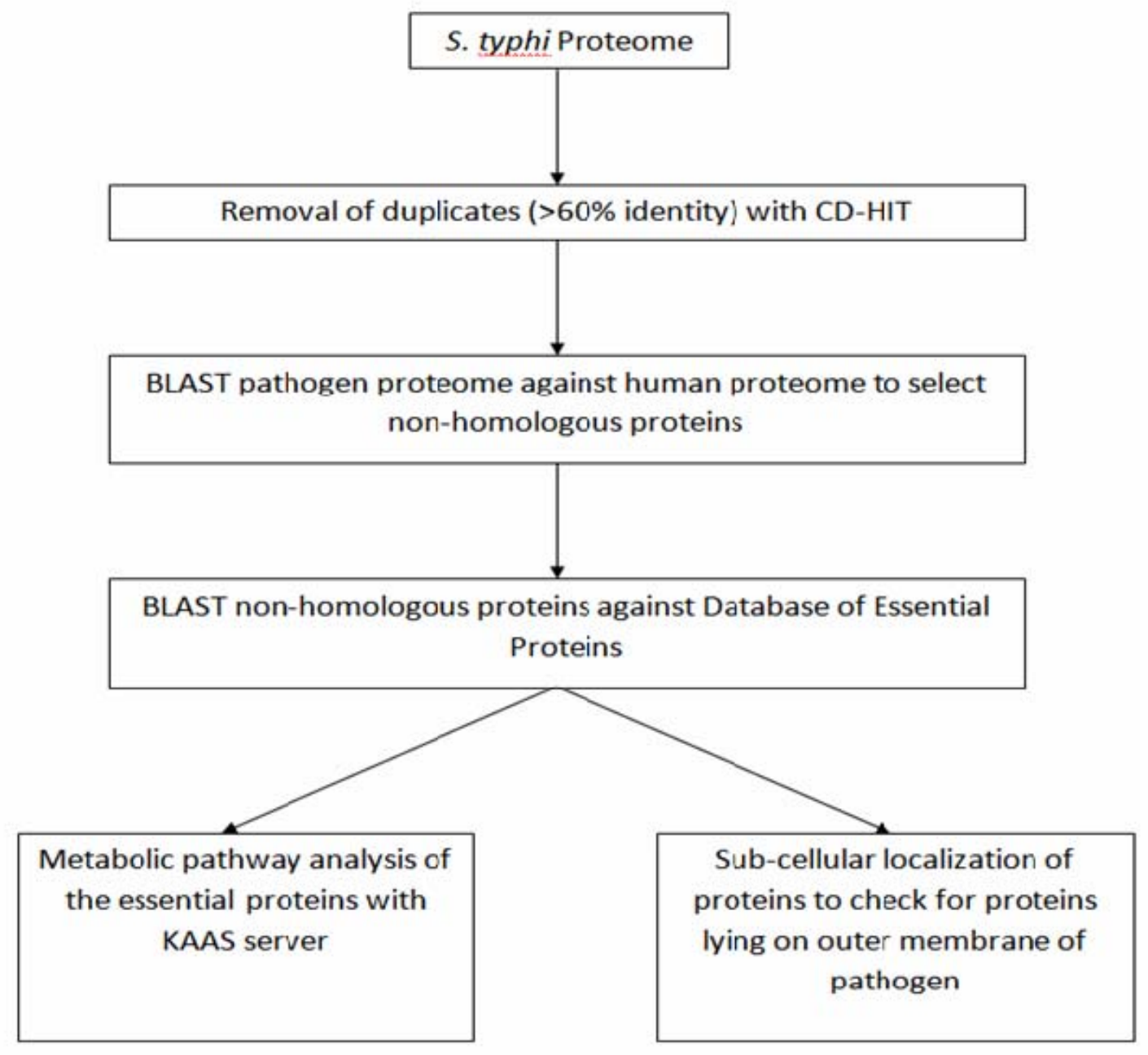

Figure 1: Flow chart for systematic identification and characterization of potential targets in salmonella typhi.

\section{Sub-cellular Localization prediction:}

Protein sub cellular localization prediction involves the computational prediction of where a protein resides in a cell. Prediction of protein sub cellular localization is an important component as it predicts the protein function and genome annotation, and it can aid the identification of targets. Sub-cellular localization analysis of the essential protein sequences has been done by Proteome Analyst Specialized Subcellular Localization Server v2.5 (PA-SUB) [16] to identify the surface membrane proteins which could be probable vaccine candidates.

\section{Discussion:}

The results obtained through computational analysis reveals that out of 4718 proteins in salmonella typhi 159 were identified as duplicates through CD-HIT with $60 \%$ similarity. The remaining 4559 paralogs were subjected to subtractive genomics which leads to 3570 proteins. These 3570 proteins when subjected to blastp against DEG database showed 300 proteins, which were essential for the pathogen. The results for subtractive proteome approach, metabolic pathway analysis and sub cellular localization are listed in Table No. 1(Supplementary material). The purpose of the present studies was to locate those essential proteins of $S$. typhi that play vital roles in the normal functioning of the bacterium within the host and to pick out them in ISSN 0973-2063 (online) 0973-8894 (print)

Bioinformation 4(4): 143-150 (2009) the view of targeting. Detection of non-human homologs in the essential proteins of $S$. typhi with subsequent screening of the proteome to find the resultant protein product are likely to lead to development of drugs that exclusively interact with the pathogen. The non-human homologs of the surface proteins would represent potential vaccine candidates. 300 of the essential proteins were without human homologs. Metabolic pathway analyses of these 300 essential proteins by KAAS server at KEGG revealed that out of 300, 149 proteins might be concluded to be unique and are invariably linked with essential metabolic and signal transduction pathways. Presumably, screening against such novel targets for functional inhibitors will result in discovery of novel therapeutic compounds active against bacteria, including the increased number of antibiotic resistant clinical strains [17].

Metabolic pathway analyses of the 149 essential proteins revealed that 15 proteins are involved in Carbohydrate Metabolism, 10 in Energy Metabolism, 5 in Lipid Metabolism, 4 in Nucleotide Metabolism, 30 in Amino Acid Metabolism, 20 in Glycan Biosynthesis and Metabolism, 16 in Metabolism of Co-factors and Vitamins, 20 in genetic information processing, 26 in environmental information processing and 2 in human disease. The results are summarized in Table 2 (Supplementary material). Comparative 144

(C) 2009 Biomedical Informatics 


\section{Bioinformation}

analysis of the metabolic pathways of the host (Homo sapiens) and the pathogen (S.typhi) by using Kyoto Encyclopedia of Genes and Genomes (KEGG) reveals 8 pathways which are unique to S.typhi. Thereafter, each selected pathway was screened for the unique enzymes and proteins involved. The peptidoglycan layer of the bacterial cell wall is the major structural element which plays an important role in pathogenesis as it provides resistance to osmotic lysis. D-alanine is the central molecule in the peptidoglycan assembly and cross-linking. D-alanine-D-alanine ligase (ddlA) is an important target as it is involved in D-alanine metabolism. Lipopolysaccharides (LPS) are also one of the main constituents of the outer cell wall of gram negative bacteria and play an important role for the survival of the pathogen. Out of the 14 enzymes involved in LPS biosyntheseis pathway, 13 enzymes are found to be essential for the variability of the bacteria and could be probable drug targets and it did not show homology with any human protein.

Two-component systems of bacteria represent the primary signal transduction paradigm in prokaryotic organisms. 8 essential enzymes were found to be potential targets in this pathway. Tryptophan synthase beta chain (trpB) is an important enzyme as it is involved in tyrosine and tryptophan biosynthesis pathway. Chemotaxis protein (MotA) and chemotaxis protein methyltransferase (CheR) is essential enzyme due to its involvement in multiple metabolic pathways like cell Motility, bacterial chemotaxis and flagellar assembly. Phosphoenolpyruvate (ppc) has been identified as a possible target due to its involvement in carbon fixation in photosynthetic organism, pyruvate metabolism and reductive carboxylase cycle. The focus of the present studies was to hunt for potential targets in $S$. typhi by computational approach. The sub-cellular localization prediction done by PA-SUB identify 11 proteins lying on the surface of the pathogen which could represent promising candidates for further characterization and analysis with a support to vaccine design. The results are summarized in Table No. 3 (Supplementary material)

\section{Conclusion:}

The availability of full genomic and proteomic sequences generated from the sequencing projects along with the computer-aided softwares to identify and characterize probable drug targets is a new emerging trend in pharmacogenomics. The application of the Database of essential genes helps to identify the potential drug targets in pathogens. The current study helps in the characterization of the potential proteins that could be targets for efficient drug design against Salmonella typhi. As subtractive genomic approach is applied for the identification of drug targets, so the drug would be specific for the pathogen and not lethal to the host. Molecular modeling of the targets will decipher the best possible active sites that can be targeted by simulations for drug design. Virtual screening against these potential targets might be useful in the discovery of potential therapeutic compounds against Salmonella typhi.

\section{References:}

[1] L Miesel et al., Nat Rev Genet. 4: 442 (2003) [PMID: 12776214]

[2] K Sakharkar et al., In Silico Biol. 4: 28 (2004) [PMID: 15724285]

[3] R Zhang et al., Nucleic Acids Res. 37: D455 (2009) [PMID: 18974178]

[4] P Everest et al.,Trends Microbiol. 9: 316 (2001) [PMID: 11435104]

[5] JE Galan Mol Microbiol. 20: 263 (1996) [PMID: 8733226]

[6] BD Jones et al., Annu Rev Immunol.14: 533 (1996) [PMID: 8717524]

[7] J Parkhill et al., Nature 413: (2001) [PMID: 11677608]

[8] B Rowe et al., Clin Infect Dis. 24: S106 (1997) [PMID: 8994789]

[9] A Kapil et al., Indian J Pathol Microbiol. 37: 179 (1994) [PMID: 7959985]

[10] A Kapil Indian J Med Res. 121: 83 (2005) [PMID: 15756040]

[11] E. Steinburg et al. Antimicrobial Resistance of Salmonella typhi in the United States: the National Antimicrobial Monitoring System (NARMS), 1999

[12] http://www.expasy.ch/sprot/

[13] http://www.ncbi.nlm.nih.gov/

[14] W Li et al., Bioinformatics 17: 282 (2001) [PMID: 11294794]

[15] Y Moriya et al., Nucleic Acids Res. 35: W182 (2007) [PMID: 17526522]

[16] Z Lu et al., Bioinformatics 20: 547 (2004) [PMID: 1499045]

[17] J Thanassi et al., Nucleic Acid Res. 30: 3152 (2002) [PMID: 12136097]

Edited by P. Kangueane

Citation: Rathi et al., Bioinformation 4(4): 143-150 (2009) License statement: This is an open-access article, which permits unrestricted use, distribution, and reproduction in any medium, for noncommercial purposes, provided the original author and source are credit. 


\section{Bioinformation}

\section{Supplementary material}

Table 1: Substractive proteomic and metabolic pathway analysis result for Salmonella typhi

\begin{tabular}{ll}
\hline Salmonella typhi & Number \\
\hline Total Number of proteins & 4718 \\
Duplicates (>60\% identical) in CD-HIT & 159 \\
Non-paralogs & 4559 \\
Non-human homologous proteins (E-value $10^{-4}$ ) & 3570 \\
Essential protein in DEG (E-value $10^{-100}$ ) & 300 \\
Essential proteins involved in metabolic pathways & 149 \\
Pathways unique to the organism (S.typhi) & 8 \\
Proteins involved in unique pathways & 27 \\
Membrane associated non-human homologs of essential genes & 11 \\
\hline
\end{tabular}

Table 2: Essential proteins of S.typhi involved in several metabolic pathways

\begin{tabular}{|c|c|c|c|c|c|}
\hline \multirow{2}{*}{\multicolumn{3}{|c|}{$\begin{array}{l}\text { SN } \quad \text { KO } \\
\text { Metabolism }\end{array}$}} & Gene Name & Pathway & EC \\
\hline & & & & & \\
\hline \multicolumn{6}{|c|}{ Carbohydrate metabolism } \\
\hline 1 & K02777 & glucose-specific IIA component & crr & $\begin{array}{l}\text { Phosphotransfe-rase } \\
\text { system }\end{array}$ & EC:2.7.1.69 \\
\hline 2 & K01643 & citrate lyase subunit alpha & citF & $\begin{array}{l}\text { Environmental } \\
\text { Information Processing }\end{array}$ & EC:4.1.3.6 \\
\hline 3 & K00117 & Quinoprotein dehydrogenase glucose & gcd & $\begin{array}{l}\text { Pentose pathway } \\
\text { phosphate }\end{array}$ & EC:1.1.1.130 \\
\hline 4 & K08092 & 3-dehydro-L-gulonate 2-Dehydrogenase & E1.1.1.130 & $\begin{array}{l}\text { Pentose and glucuronate } \\
\text { Interconversions }\end{array}$ & EC:1.1.1.130 \\
\hline 5 & K02798 & mannitol-specific IIA component & mtlA & $\begin{array}{l}\text { Phosphotransfe-rase } \\
\text { system }\end{array}$ & EC:2.7.1.69 \\
\hline 6 & K01818 & L-fucose isomerase & fucI & $\begin{array}{l}\text { Fructose and mannose } \\
\text { metabolism }\end{array}$ & EC 5.3.1.25 \\
\hline 7 & K02821 & ascorbate-specific IIA component & sgaA & $\begin{array}{l}\text { Phosphotransfe-rase } \\
\text { system }\end{array}$ & EC:2.7.1.69 \\
\hline 8 & K01788 & $\begin{array}{l}\text {-acylglucosamine-6- } \\
\text { phosphate 2-epimerase }\end{array}$ & nanE & Aminosugars metabolism & EC:5.1.3.9 \\
\hline 9 & K03431 & phosphoglucosamine mutase & glmM & Aminosugars metabolism & EC:5.4.2.10 \\
\hline 10 & K00790 & $\begin{array}{l}\text { UDP-N-acetylglucosamine } 1 \text { - } \\
\text { carboxyvinyltransferase }\end{array}$ & murA & Aminosugars metabolism & EC:2.5.1.7 \\
\hline 11 & K00075 & $\begin{array}{l}\text { UDP-N-acetylmuramate } \\
\text { dehydrogenase }\end{array}$ & murB & Aminosugars metabolism & EC:1.1.1.158 \\
\hline 12 & K01595 & $\begin{array}{l}\text { phosphoenolpyruvate } \\
\text { carboxylase }\end{array}$ & Ppc & Pyruvate metabolism & EC:4.1.1.31 \\
\hline 13 & K00656 & formate C-acetyltransferase & pflD & Pyruvate metabolism & EC:2.3.1.54 \\
\hline 14 & K00925 & acetate kinase & ackA & Pyruvate metabolism & EC:2.7.2.1 \\
\hline 15 & K00932 & propionate kinase & $\operatorname{tdcD}$ & Propanoate metabolism & EC:2.7.2.15 \\
\hline \multicolumn{6}{|c|}{ Energy metabolism } \\
\hline 1 & K00425 & cytochrome bd-I oxidase subunit I & cydA & $\begin{array}{l}\text { Oxidative } \\
\text { phosphorylation }\end{array}$ & EC:1.10.3 \\
\hline 2 & K00426 & cytochrome bd-I oxidase subunit I & cydB & $\begin{array}{l}\text { Oxidative } \\
\text { phosphorylation }\end{array}$ & EC:1.10.3 \\
\hline 3 & K01595 & $\begin{array}{l}\text { phosphoenolpyruvate } \\
\text { carboxylase }\end{array}$ & Ppc & Pyruvate metabolism & EC:4.1.1.31 \\
\hline 4 & K00926 & carbamate kinase & arc & Nitrogen metabolism & EC:2.7.2.2 \\
\hline 5 & K01916 & NAD+ synthase & NadE & Nitrogen metabolism & EC:6.3.1.5 \\
\hline 6 & K01914 & aspartate--ammonia ligase & Asn A & Nitrogen metabolism & EC:6.3.1.1 \\
\hline 7 & K00264 & Glutamate synthase (NADPH/NADH) & GLT1 & Nitrogen metabolism & $\begin{array}{l}\text { EC:1.4.1.13 } \\
1.4 .1 .14\end{array}$ \\
\hline 8 & K03385 & formate-dependent nitrite reductase & NrfA & Nitrogen metabolism & EC:1.7.2.2 \\
\hline 9 & K00369 & nitrate reductase & E1.7.99.4 & Nitrogen metabolism & EC:1.7.99.4 \\
\hline 10 & K00640 & serine O-acetyltransferase & CysE & Sulfur metabolism & EC:2.3.1.30 \\
\hline \multicolumn{6}{|c|}{ Lipid metabolism } \\
\hline 1 & K00648 & 3-oxoacyl-[acyl-carrier-protein] synthase III & fabH & Fatty acid biosynthesis & EC:2.3.1.180 \\
\hline 2 & K03527 & 4-hydroxy-3-methylbut-2-enyl diphosphate reductase & $\mathrm{ispH}$ & Biosynthesis of steroids & EC:1.17.1.2 \\
\hline 3 & K03526 & (E)-4-hydroxy-3-methylbut- 2-enyl-diphosphate synthase & ispG & Biosynthesis of steroids & EC:1.17.7.1 \\
\hline 4 & K00919 & 4-diphosphocytidyl-2-C-methyl-D-erythritol kinase & ispE & Biosynthesis of steroids & EC:2.7.1.148 \\
\hline 5 & K00099 & $\begin{array}{l}\text { 1-deoxy-D-xylulose-5- } \\
\text { phosphate reductoisomerase }\end{array}$ & Dxr & Biosynthesis of steroids & EC:1.1.1.267 \\
\hline
\end{tabular}


Nucleotide metabolism

2 K09903 uridylate kinase

3 K03040 DNA-directed RNA polymerase subunit alpha

$4 \quad$ K02319 DNA polymerase I

Amino acid metabolism

$1 \quad$ K00926 carbamate kinase

2 K01776 glutamate racemase

3 K01775 alanine racemase

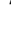

10

11

24 K01695 Tryptophan synthase alpha

$$
\text { chain }
$$

25 K01736 chorismate synthase

26 K01850 chorismate mutase

27 K00145 N-acetyl-gamma-glutamyl- phosphate reductase

Metabolism of another amino acids

1 K01925 UDP-N-acetylmuramoylalanine--D-

2 glutamate ligase

2 K01924 UDP-N-acetylmuramate-alanine ligase

3 K01921 D-alanine-D-alanine ligase

Glycan biosynthesis and metabolism

1 K00677 UDP-N-acetylglucosamine

ISSN 0973-2063 (online) 0973-8894 (print)

Bioinformation 4(4): 143-150 (2009)
relA
pyrH

pyrH

rpoA

polB1

Purine metabolism

Pyrimidine metabolism

$\mathrm{EC}: 2.7 .6 .5$

Genetic Information

Processing

Purine metabolism

EC:2.7.7.6

$\mathrm{EC}: 2.7 .7 .7$

arc

murI

Alr

pepD

thrA

asd

Glutamate metabolism Glutamate metabolism

Alanine and aspartate

metabolism

Alanine and aspartate

metabolism

Glycine,serine and

threonine metabolism

Glycine,serine and

threonine metabolism

metE Methionine metabolism

mtnN, mtn,pfs Methionine metabolism

EC:2.7.2.2

EC:5.1.1.3

EC:5.1.1.1

EC:3.4.13.3

EC:1.1.1.3

EC:1.2.1.11

EC:2.1.1.14

EC:3.2.2.9

dapB Lysine biosynthesis

dapD Lysine biosynthesis

EC:1.3.1.26

EC:2.3.1.117

dapF Lysine biosynthesis

murF

Lysine biosynthesis

EC:5.1.1.7

EC:6.3.2.10

murE

Lysine biosynthesis

EC:6.3.2.13

astB

Arginine and proline

EC:3.5.3.23

astA

metabolism

Arginine and proline metabolism

hisG

hisE

hisI

hisB

hisB

aroF,aroG,

aroH

ARO1

Histidine metabolism

Histidine metabolism

Histidine metabolism

Histidine metabolism

Histidine metabolism

Phenylalanine, tyrosine and tryptophan

biosynthesis

Phenylalanine, tyrosine

and tryptophan

biosynthesis

trpB Phenylalanine, tyrosine

and tryptophan

biosynthesis

trpA Phenylalanine, tyrosine

and tryptophan

biosynthesis

aroC Phenylalanine, tyrosine

and tryptophan

biosynthesis

E5.4.99.5 Phenylalanine, tyrosine

and tryptophan

biosynthesis

$\arg \mathrm{C}$

Urea cycle And

metabolism of amino

groups

EC:2.3.1.109

EC:2.4.2.17

EC:3.6.1.31

EC:3.5.4.19

EC:4.2.1.19

EC:4.2.1.19

3.1.3.15

EC:2.5.1.54

EC:4.2.3.4

EC:4.2.1.20

EC:4.2.1.20

EC:4.2.3.5

EC:5.4.99.5

EC:1.2.1.38

$\begin{array}{ll}\text { murD } & \text { D-Glutamine and } \\ \text { D-glutamate metabolism }\end{array}$

murC D-Glutamine and

EC:6.3.2.9

D-glutamate metabolism

ddlA D-Alanine metabolism

EC:6.3.2.8

EC:6.3.2.4

$\operatorname{lpxA}$

Lipopolysaccharide

EC:2.3.1.129 


\begin{tabular}{|c|c|c|c|c|c|}
\hline & & acyltransferase & & biosynthesis & \\
\hline 2 & K02535 & $\begin{array}{l}\text { UDP-3-O-[3-hdroxymyristoyl] } \\
\text { deacetylase }\end{array}$ & lpxC & $\begin{array}{l}\text { Lipopolysaccharide } \\
\text { biosynthesis }\end{array}$ & EC:3.5.1. \\
\hline 3 & K02536 & $\begin{array}{l}\text { UDP-3-O-[3- hydroxymyristoyl] } \\
\text { glucosamine N-acyltransferase }\end{array}$ & lpxD & $\begin{array}{l}\text { Lipopolysaccharide } \\
\text { biosynthesis }\end{array}$ & EC:2.3.1. \\
\hline 4 & K03269 & $\begin{array}{l}\text { UDP-2,3-diacylglucosamine } \\
\text { hydrolase }\end{array}$ & lpxH & $\begin{array}{l}\text { Lipopolysaccharide } \\
\text { biosynthesis }\end{array}$ & EC:3.6.1. \\
\hline 5 & K00748 & lipid-A-disaccharide synthase & $\operatorname{lpxB}$ & $\begin{array}{l}\text { Lipopolysaccharide } \\
\text { biosynthesis }\end{array}$ & EC:2.4.1.182 \\
\hline 6 & K00912 & tetraacyldisaccharide 4'-kinase & lpxK & $\begin{array}{l}\text { Lipopolysaccharide } \\
\text { biosynthesis }\end{array}$ & EC:2.7.1.130 \\
\hline 7 & K02527 & 3-deoxy-D-manno-octulosonic-acid transferase & kdtA & $\begin{array}{l}\text { Lipopolysaccharide } \\
\text { biosynthesis }\end{array}$ & EC:2.-.-.- \\
\hline 8 & K00979 & 3-deoxy-manno-octulosonate cytidylyltransferase & kdsB & $\begin{array}{l}\text { Lipopolysaccharide } \\
\text { biosynthesis }\end{array}$ & EC:2.7.7.38 \\
\hline 9 & K01627 & 2-dehydro-3-deoxyphosphooctonate aldolase & kdsA & $\begin{array}{l}\text { Lipopolysaccharide } \\
\text { biosynthesis }\end{array}$ & EC:2.5.1.55 \\
\hline 10 & K02841 & heptosyltransferase I & waaC, rfaC & $\begin{array}{l}\text { Lipopolysaccharide } \\
\text { biosynthesis }\end{array}$ & EC:2.4.-.- \\
\hline 11 & K02843 & heptosyltransferase II & waaF, rfaF & $\begin{array}{l}\text { Lipopolysaccharide } \\
\text { biosynthesis }\end{array}$ & EC:2.4.-.- \\
\hline 12 & K02840 & Galactosyltransferase & waaB, rfaB & $\begin{array}{l}\text { Lipopolysaccharide } \\
\text { biosynthesis }\end{array}$ & EC:2.4.1.- \\
\hline 13 & K02844 & Glucosyltransferase & waaG, rfaG & $\begin{array}{l}\text { Lipopolysaccharide } \\
\text { biosynthesis }\end{array}$ & EC:2.4.1.- \\
\hline 14 & K02847 & O-antigen ligase & waaL, rfaL & $\begin{array}{l}\text { Lipopolysaccharide } \\
\text { biosynthesis }\end{array}$ & EC:6.-.-.- \\
\hline 15 & K01921 & D-alanine-D-alanine ligase & ddlA & $\begin{array}{l}\text { Peptidoglycan } \\
\text { biosynthesis }\end{array}$ & EC:6.3.2.4 \\
\hline 16 & K01000 & phospho-N-acetylmuramoyl-pentapeptide-transferase & mraY & $\begin{array}{l}\text { Peptidoglycan } \\
\text { biosynthesis }\end{array}$ & EC:2.7.8.13 \\
\hline 17 & K02563 & $\begin{array}{l}\text { UDP-N-acetylglucosamine--N-acetylmuramyl- } \\
\text { (pentapeptide) pyrophosphoryl-undecaprenol } \\
\text { N-acetylglucosamine transferase }\end{array}$ & murG & $\begin{array}{l}\text { Peptidoglycan } \\
\text { biosynthesis }\end{array}$ & EC:2.4.1.227 \\
\hline 18 & K01924 & UDP-N-acetylmuramate-alanine ligase & murC & $\begin{array}{l}\text { Peptidoglycan } \\
\text { biosynthesis }\end{array}$ & EC:6.3.2.8 \\
\hline 19 & K01925 & UDP-N-acetylmuramoylalanine--D-glutamate ligase & murD & $\begin{array}{l}\text { Peptidoglycan } \\
\text { biosynthesis }\end{array}$ & EC:6.3.2.9 \\
\hline 20 & K03587 & cell division protein FtsI & $\mathrm{ftsI}$ & $\begin{array}{l}\text { Peptidoglycan } \\
\text { biosynthesis }\end{array}$ & EC:2.4.1.129 \\
\hline \multicolumn{6}{|c|}{ Metabolism of Co-factors and Vitamins } \\
\hline 1 & K03147 & thiamine biosynthesis protein ThiC & thiC & Thiamine metabolism & \\
\hline 2 & K00946 & thiamine-monophosphate kinase & thiL & Thiamine metabolism & EC:2.7.4.16 \\
\hline 3 & K01497 & GTP cyclohydrolase II & ribA & Riboflavin metabolism & EC:3.5.4.25 \\
\hline 4 & K01498 & diaminohydroxyphosphoribosylaminopyrimidine deaminase & E3.5.4.26 & Riboflavin metabolism & EC:3.5.4.26 \\
\hline 5 & K00082 & 5-amino-6-(5-phosphoribosylamino) uracil reductase & E1.1.1.193 & Riboflavin metabolism & EC:1.1.1.193 \\
\hline 6 & K02858 & 3,4-dihydroxy 2-butanone 4-phosphate synthase & ribB & Riboflavin metabolism & \\
\hline 7 & K00793 & riboflavin synthase alpha chain & ribE & Riboflavin metabolism & EC:2.5.1.9 \\
\hline 8 & K03474 & pyridoxine synthase 5-phosphate & pdxJ & Riboflavin metabolism & EC:2.6.99.2 \\
\hline 9 & K00969 & nicotinate-nucleotide adenylyltransferase & nadD & $\begin{array}{l}\text { Nicotinate and } \\
\text { nicotinamide metabolism }\end{array}$ & EC:2.7.7.18 \\
\hline 10 & K03517 & quinolinate synthase & nadA & $\begin{array}{l}\text { Nicotinate and } \\
\text { nicotinamide metabolism }\end{array}$ & \\
\hline 11 & K01012 & biotin synthetase & bioB & Biotin metabolism & EC:2.8.1.6 \\
\hline 12 & K01664 & $\begin{array}{l}\text { para-aminobenzoate synthetase } \\
\text { component II }\end{array}$ & pabA & Folate biosynthesis & EC:2.6.1.85 \\
\hline 13 & K02302 & $\begin{array}{l}\text { uroporphyrin-III C- methyltransferase / precorrin-2 } \\
\text { dehydrogenase / sirohydrochlorin ferrochelatase }\end{array}$ & cysG & $\begin{array}{l}\text { Porphyrin and chlorophyll } \\
\text { metabolism }\end{array}$ & $\begin{array}{l}\text { EC:2.1.1.107 } \\
\text { 1.3.1.76 } \\
4.99 .1 .4\end{array}$ \\
\hline 14 & K02492 & glutamyl-tRNA reductase & hemA & $\begin{array}{l}\text { Porphyrin and chlorophyll } \\
\text { metabolism }\end{array}$ & EC:1.2.1.70 \\
\hline 15 & K02551 & $\begin{array}{l}\text { 2-succinyl-5-enolpyruvyl-6-hydroxy-3-cyclohexene-1- } \\
\text { carboxylate synthase }\end{array}$ & mend & $\begin{array}{l}\text { Ubiquinone and } \\
\text { menaquinone biosynthesis }\end{array}$ & EC:2.2.1.9 \\
\hline 16 & K03182 & $\begin{array}{l}\text { 3-octaprenyl-4-hydroxybenzoate } \\
\text { carboxy- lyase UbiD }\end{array}$ & ubiD & $\begin{array}{l}\text { Ubiquinone and } \\
\text { menaquinone biosynthesis }\end{array}$ & EC:4.1.1.- \\
\hline
\end{tabular}

Xenobiotics Biodegradation and Metabolism 


\section{Bioinformation}

\section{Genetic information processing \\ Transcription \\ DNA-directed RNA polymerase subunit alpha \\ Translation \\ $1 \quad$ K02986 \\ 2 K01878 glycyl-tRNA synthetase alpha chain \\ Folding, sorting and degradation \\ $1 \quad$ K03070 Preprotein translocase SecA subunit \\ 2 K03076 preprotein translocase SecY subunit \\ $3 \quad$ K03072 Preprotein translocase SecD subunit \\ $4 \quad$ K03074 preprotein translocase SecF subunit \\ Replication and Repair

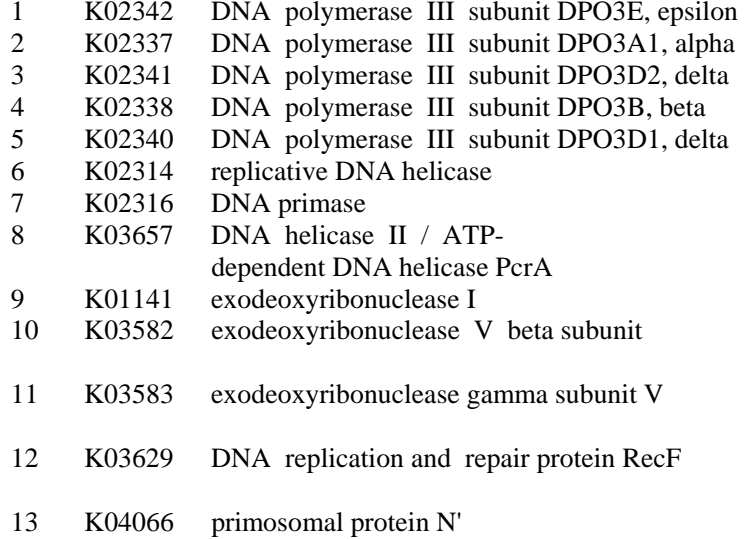

rроA

RNA polymerase

EC:2.7.7.6

Environmental Information Processing

Membrane Transport

2 K11070 spermidine/putrescine transport system permease protein $3 \quad$ K11069 spermidine/putrescine transport system substrate-binding

$4 \quad$ K10540 methyl-galactoside transport system protein

\begin{tabular}{|c|c|c|}
\hline 5 & K02040 & phosphate transport system substrate-binding protein \\
\hline 6 & K10015 & histidine transport system permease protein \\
\hline 7 & K10002 & glutamate/aspartate transport system permease protein \\
\hline 8 & K10009 & cystine transport system permease protein \\
\hline 9 & K02035 & $\begin{array}{l}\text { peptide/nickel transport system } \\
\text { substrate-binding protein }\end{array}$ \\
\hline 10 & K02016 & iron complex transport system substrate-binding protein \\
\hline 11 & K09808 & lipoprotein-releasing system permease protein \\
\hline 12 & K09811 & cell division transport system permease protein \\
\hline 13 & K07091 & lipopolysaccharide export system permease protein \\
\hline 14 & K11720 & lipopolysaccharide export system permease protein \\
\hline 15 & K02778 & PTS system, glucose-specific IIB component \\
\hline 16 & K03475 & PTS system, ascorbate-specific IIC component \\
\hline
\end{tabular}

ISSN 0973-2063 (online) 0973-8894 (print) Bioinformation 4(4): 143-150 (2009)

$\begin{array}{clr}\begin{array}{c}\text { rpsD } \\ \text { glyQ }\end{array} & \begin{array}{l}\text { Translation } \\ \text { Aminoacyl-tRNA } \\ \text { biosynthesis }\end{array} & \\ \text { secA } & \begin{array}{l}\text { Folding, Sorting and } \\ \text { Degradation }\end{array} \\ \text { secY } & \begin{array}{l}\text { Folding, Sorting and } \\ \text { Degradation } \\ \text { secD }\end{array} & \begin{array}{l}\text { Folding, Sorting and } \\ \text { Degradation }\end{array} \\ \text { secF } & \begin{array}{l}\text { Folding, Sorting and } \\ \text { Degradation }\end{array}\end{array}$

EC:6.1.1.14

$\begin{array}{lll}\text { dnaQ } & \text { DNA replication } & \text { EC:2.7.7.7 } \\ \text { dnaE } & \text { DNA Replication } & \text { EC:2.7.7.7 } \\ \text { holB } & \text { DNA Replication } & \text { EC:2.7.7.7 } \\ \text { dnaN } & \text { DNA Replication } & \text { EC:2.7.7.7 } \\ \text { holA } & \text { DNA Replication } & \text { EC:2.7.7.7 } \\ \text { dnaB } & \text { DNA Replication } & \text { EC:3.6.1.- } \\ \text { dnaG } & \text { DNA Replication } & \text { EC:2.7.7.- } \\ \text { uvrD, pcrA } & \text { Nucleotide excision repair } & \text { EC:3.6.1.- } \\ \text { sbcB } & \text { Mismatch repair } & \text { EC:3.1.11.1 } \\ \text { recB } & \begin{array}{l}\text { Homologous } \\ \text { recombination }\end{array} & \text { EC:3.1.1.5 } \\ \text { recC } & \begin{array}{l}\text { Homologous } \\ \text { recombination }\end{array} & \text { EC:3.1.11.5 } \\ \text { recF } & \text { Homologous } & \text { EC:3.1.11.5 } \\ \text { priA } & \text { recombination } & \\ & \text { Homologous } & \text { EC:3.6.1.- }\end{array}$

cysW ABC transporters

potC ABC Transporters

potD ABC transporters

mglB ABC Transporters

pstS

ABC Transporters

hisM ABC Transporters

gltK ABC Transporters

ABC.CYST.P ABC Transporters

ABC.PE.S ABC Transporters

ABC.FEV.S ABC Transporters

ABC.LPT.P, ABC Transporters

lolC, lolE

ftsX

lptF

lptG

ABC Transporters

ABC Transporters

ABC transporters

PTS-Glc-

EIIB, ptsG

PTS-Ula-

EIIC, laA,

sgaT
EC:2 7.7.7

$\mathrm{EC}: 3.6 .1$.

EC:2.7.7.-

C:3.1.11.1 


\section{Bioinformation}

\begin{tabular}{|c|c|c|c|c|c|}
\hline 17 & K08484 & phosphotransferase system, enzyme I, PtsP & $\begin{array}{l}\text { PTS- } \\
\text { EI.PTSP, } \\
\text { ptsP }\end{array}$ & & \\
\hline \multicolumn{6}{|c|}{ Signal Transduction } \\
\hline 1 & K07636 & $\begin{array}{l}\text { two-component system, OmpR family, phosphate regulon } \\
\text { sensor histidine kinase PhoR }\end{array}$ & PhoR & Signal Transduction & EC:2.7.13.3 \\
\hline 2 & K07639 & $\begin{array}{l}\text { two-component system, OmpR family, sensor histidine } \\
\text { kinase RstB }\end{array}$ & RstB & Signal Transduction & EC:2.7.13.3 \\
\hline 4 & K00370 & nitrate reductase 1 , alpha subunit & narG & Signal Transduction & EC:1.7.99.4 \\
\hline 5 & K00990 & [protein-PII] uridylyltransferase & $\operatorname{gln} \mathrm{D}$ & Signal Transduction & EC:2.7.7.59 \\
\hline 6 & K03407 & $\begin{array}{l}\text { two-component system, chemotaxis family, sensor kinase } \\
\text { CheA }\end{array}$ & cheA & Signal Transduction & EC:2.7.13.3 \\
\hline 7 & K00575 & chemotaxis protein methyltransferase CheR & cheR & Signal Transduction & EC:2.1.1.80 \\
\hline \multicolumn{6}{|c|}{ Human Diseases } \\
\hline 1 & K03092 & RNA polymerase sigma-54 factor & SIG54, rpoN & $\begin{array}{l}\text { Vibrio cholerae } \\
\text { phathogenic cycle }\end{array}$ & \\
\hline 2 & K05851 & adenylate cyclase, class 1 & $\begin{array}{l}\text { E4.6.1.1A, } \\
\text { cyaA }\end{array}$ & $\begin{array}{l}\text { Vibrio cholerae } \\
\text { phathogenic cycle }\end{array}$ & EC:4.6.1.1 \\
\hline
\end{tabular}

Table 3: List of the outer membrane proteins of Salmonlla typhi identified by PA-SUB

\begin{tabular}{llll}
\hline S.N & Accession No & Name of Protein & Sub-Cellular Localization \\
\hline 1 & Q56110 & Outer membrane prorein S1 & Outer membrane \\
2 & Q56119 & Outer membrane pore protein & Outer membrane \\
3 & Q8Z8P3 & Outer membrane usher protein FimD & Outer membrane \\
4 & Q8Z944 & Outer membrane fimbrial usher protein & Outer membrane \\
5 & Q8Z4Y8 & Long chain fatty acid transport protein & Outer membrane \\
6 & Q8Z1S4 & Putative Type-I section protein & Outer membrane \\
7 & Q8XEL5 & Putative exported protein & Outer membrane \\
8 & Q8Z9A3 & Outer membrane protein assembly factor yaeT & Outer membrane \\
9 & Q8Z9J6 & LPS-assembly protein & Outer membrane \\
10 & Q8Z4J0 & Putative lipoprotein & Outer membrane \\
11 & Q8Z6A0 & Outer membrane lipoprotein lolB & Outer membrane \\
\hline
\end{tabular}

